\title{
Influência da granulometria da matéria-prima na expansão de extrusados de milho
} Influence of raw material particle size on the expansion of extruded corn

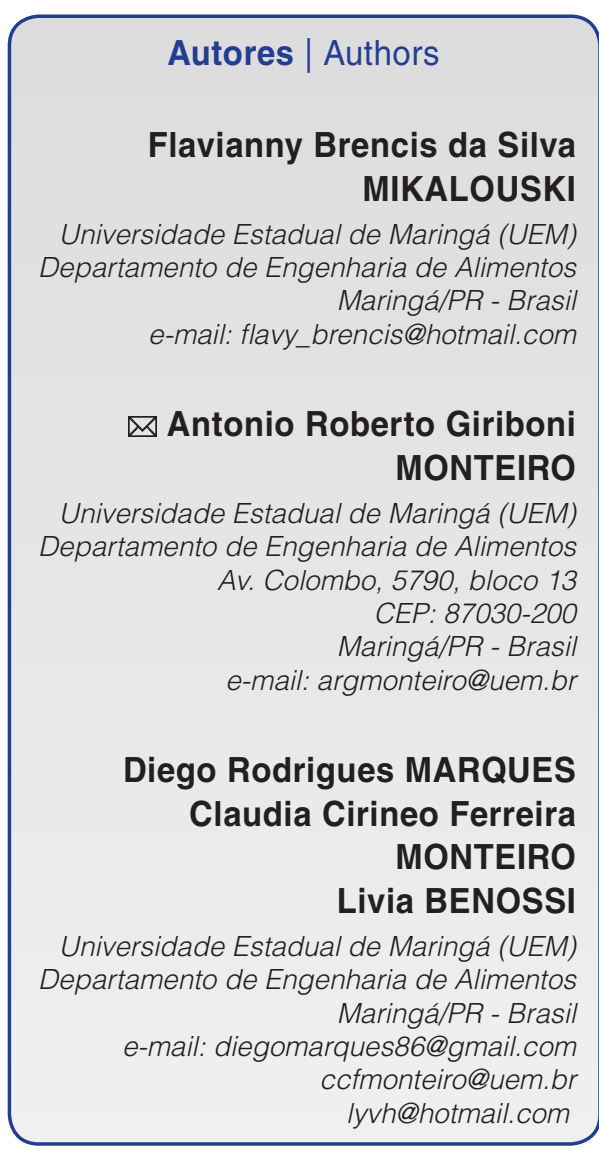

Autor Correspondente / Corresponding Author

Recebido / Received: 27/05/2013 Aprovado / Approved: 25/02/2014 Publicado / Published: mar./2014

\section{Resumo}

$\mathrm{Na}$ produção de extrusados de milho, a indústria usualmente emprega o grits, que consiste no milho limpo, degerminado e moído em granulometria média. Entretanto, visando à redução dos custos de produção, fabricantes de snacks extrusados de milho vêm utilizando o fubá em substituição ao grits, devido ao menor custo apresentado por este. O objetivo deste trabalho foi verificar a influência da granulometria do milho sobre o índice de expansão dos snacks, sua textura e aceitação sensorial. Para isso, foram utilizadas três diferentes granulometrias do milho moído, sendo: grits, fubá mimoso e fubá mimoso fino. O índice de expansão radial foi analisado pela razão entre o diâmetro médio do snack e o diâmetro da matriz utilizada, a textura foi analisada em texturômetro, modelo Texture Analyser TA-XT Plus (Stable Micro Systens). A partir dos resultados obtidos, houve a confirmação da influência da granulometria nos parâmetros físicos, entretanto, não ocorreu alteração na aceitação do produto, indicando, desta forma, ser viável a substituição do grits pelo fubá na produção de snacks sob o ponto de vista do produto final.

Palavras-chave: Extrusão; Milho; Granulometria.

\section{Summary}

In order to produce extruded corn snacks, industry has usually employed grits (clean, degerminated corn, milled to a medium particle size). However, aiming at reducing production costs, extruded corn snack producers have been replacing grits by corn flour due to lower costs. The objective of this study was to assess the influence of corn particle size on the snack expansion rate, texture and sensory acceptance. Thus, three different milled corn particle sizes were used in this study: grits, mimoso corn flour and fine mimoso corn flour. The expansion rate was analyzed from the ratio between the mean snack diameter and the diameter of the matrix used, and the texture was analyzed using aTA-XT Plus Texture Analyser (Stable Micro Systems) with the WB-12 probe. The results obtained proved the influence of particle size on the physical parameters, but there was no change in product acceptance, indicating the feasibility of replacing grits by corn flour to produce snacks, when considering the final product.

Key words: Extrusion; Corn; Particle size. 


\section{Introdução}

Para que o milho seja utilizado como matéria-prima na indústria de extrusados, é necessária a redução dos grãos por moagem. Na moagem via úmida, ocorre a separação dos componentes e obtenção de amido, proteínas, fibras e gérmen. No processo de moagem via seca, são obtidas diferentes frações granulométricas, como canjicão, canjica, grits, fubá e farinha de milho (SCHMIELE, 2009).

De acordo com Camargo et al. (1984), o fubá comum é obtido pela moagem do milho integral, possuindo as mesmas composições. Já o fubá mimoso e o grits são produzidos pelo grão limpo e degerminado, com isso, tanto grits como fubá mimoso apresentam composições iguais, possuindo grande quantidade de carboidratos, em especial amido, e baixas quantidades de proteínas, lipídios e minerais. Uma importante aplicação do milho na indústria de alimentos é o uso do grão degerminado e moído para a produção de alimentos extrusados conhecidos como snacks (GONÇALVES et al., 2003).

A tecnologia de extrusão, nos últimos tempos, tem se tornado um dos principais processos no desenvolvimento de produtos alimentícios. A extrusão é um processo contínuo, no qual a matéria-prima é forçada a partir de uma matriz ou molde, em condições de mistura e aquecimento, pressão e fricção, que levam à gelatinização do amido, à desnaturação de proteínas e à ruptura de pontes de hidrogênio (THAKUR e SAXENA, 2000).

A extrusão termoplástica apresenta várias vantagens sobre os processos convencionais como menor tempo e custo de processo, menor espaço físico necessário e maior flexibilidade para a produção de produtos variados (OLIVEIRA et al., 2013).

Este trabalho teve por objetivo avaliar o efeito da granulometria de diferentes produtos de milho sobre a expansão, textura e análise sensorial de snacks extrusados.

\section{Material e métodos}

\subsection{Matéria-prima}

Os snacks foram preparados a partir de três diferentes matérias-primas: grits de milho, fubá mimoso e fubá mimoso fino, os quais foram obtidos junto à empresa Kowalski Alimentos, Apucarana, PR.

\subsection{Análise granulométrica}

As matérias-primas para produção dos snacks foram caracterizadas quanto à sua granulometria, segundo metodologia descrita pela Abimilho (2003), em duplicata. Uma fração de 100 gramas de cada amostra foi depositada em um sistema de peneiras sobrepostas (30, 40, 50 e 60 mesh - ABNT, respectivamente 0,6; 0,425; 0,3; $0,25 \mathrm{~mm}$ ) e submetidas à agitação até obtenção de uma massa constante do material retido em cada peneira. Os resultados estão expressos em percentagem.

\subsection{Determinação de amido}

A determinação de amido foi efetuada nas três amostras de matéria-prima, em duplicata, a partir do método polarimétrico. Foram pesados 2,5 g de amostra, a qual foi hidrolisada com adição de ácido clorídrico $1,128 \%$ com aquecimento em banho-maria e posterior resfriamento em banho de gelo. Foram adicionados acetato de zinco e ferrocianeto de potássio, para clarificação (ABIMILHO, 2003). Depois da filtração para eliminação da turbidez, as amostras foram passadas pelo sacarímetro Bellingham+Stanley Limited (London).

A obtenção da percentagem de amido nas amostras foi efetuada a partir da Equação 1.

$\%$ Amido $=\frac{691 \times\left(\mathrm{P}-\mathrm{P}^{\prime}\right)}{\alpha \times \mathrm{D} 20^{\circ}}$

$\mathrm{P}=$ leitura da determinação da amostra; $\mathrm{P}^{\prime}=$ leitura da prova em branco $(2,75) ; \alpha \times \mathrm{D}_{2} 0^{\circ}=$ fator específico para cada tipo de amido (fontes de milho $=187,6$ ).

\subsection{Processamento dos snacks}

A extrusão foi efetuada no equipamento IMBRA RX50 (INBRAMAQ, Ribeirão Preto, SP, Brasil), com rosca única de $50 \mathrm{~mm}$ de diâmetro e $200 \mathrm{~mm}$ de comprimento. A matriz utilizada possuía dois furos de $3 \mathrm{~mm}$ de diâmetro e os parâmetros foram fixados com a amperagem do motor em $20 \mathrm{~A}$ e a alimentação dos sistema em $15 \mathrm{~g} / \mathrm{s}$. O corte foi realizado a $120 \mathrm{rpm}$.

Foram processados 500 gramas de cada amostra com duas repetições e, posteriormente, homogeneizadas e secas em tambor rotativo a $60^{\circ} \mathrm{C}$.

As amostras foram pré-condicionadas a uma umidade de $20 \%$, antes do processo, que ocorreu de forma constante, com as três amostras consecutivas, sem que houvesse alteração nos parâmetros da extrusora.

Depois da extrusão, os snacks foram submetidos ao processo de secagem por um período de 3 horas a $60{ }^{\circ} \mathrm{C}$ e, em seguida, foram para a drageadeira e pulverizados com $8 \%$ de óleo vegetal de soja (Soya, Brasil), aromatizados com uma mistura contendo 2,0\% de condimento preparado de ervas finas (All-Flavors, Brasil) e 2,0\% de aji-sal (Ajinomoto, Brasil). A massa dos ingredientes adicionados foram calculados em relação à massa do extrusado. 


\section{5 Índice de expansão radial}

$O$ índice de expansão radial (IE) foi calculado segundo Mercier et al. (1998), pela razão entre o diâmetro médio do extrusado e o diâmetro da matriz do extrusor, em 10 diferentes produtos expandidos, para cada tratamento. O diâmetro foi medido utilizando-se paquímetro digital (Marberg, Mb-300, São Paulo). O cálculo do (IE) está representado na Equação 2.

$$
\mathrm{IE}=\frac{\text { Diâmetro da amostra }}{\text { Diâmetro da matriz }}
$$

\subsection{Análise de textura}

A análise de textura mecânica foi realizada no Texture Analyser TAXT2 Plus (Stable Micro Systems, Inglaterra), de acordo com Dischsen et al. (2013), no qual as amostras foram dispostas horizontalmente sobre a plataforma e foi utilizado o probe Warner Bratzler de $12 \times 7 \mathrm{~cm}(\mathrm{HDP} / \mathrm{BS})$, com carga máxima de $5 \mathrm{~kg}$, que rompeu a amostra como uma guilhotina. Os resultados foram expressos em quilogramas e representam a média aritmética de 10 determinações de força de ruptura para amostras provenientes de um mesmo ensaio. Os parâmetros utilizados nos testes foram: (i) velocidade pré-teste $=1,5 \mathrm{~m} / \mathrm{s}$; (ii) velocidade de teste $=2,0 \mathrm{~m} / \mathrm{s}$; (iii) velocidade pós-teste $=10,0 \mathrm{~m} / \mathrm{s}$; (iv) força $=0,20 \mathrm{~N}$; (v) ciclo de contagem = 5 segundos; (vi) sensibilidade do aparelho $=15 \mathrm{~g}$, com medida de força em compressão.

\subsection{Análise sensorial}

Os testes sensoriais foram realizados no Laboratório de Análise Sensorial do Departamento de Engenharia de Alimentos da Universidade Estadual de Maringá, com 50 provadores não treinados. A avaliação consistiu em um teste de ordenação-preferência, na qual o provador ordena os produtos a partir do que menos gostou até o que mais gostou. A seguir, são atribuídas notas crescentes (de 1 a 3) em função da posição apontada por cada provador (MONTEIRO e CESTARI, 2013). Neste teste, cinco unidades de cada uma das amostras, (1), (2) e (3), foram apresentadas em copos descartáveis, devidamente identificadas com números aleatórios de três dígitos, acompanhadas de um copo de água. Nas cabines de teste, foi utilizada lâmpada de luz laranja, para que a coloração dos produtos não influenciasse a escolha de preferência. Os testes sensoriais tiveram aprovação pelo Comitê de Ética em Pesquisa da Universidade Estadual de Maringá (CAAE 18718013.3.0000.0104).

\subsection{Análise estatística}

Os dados obtidos foram avaliados estatisticamente a partir da análise de variância (ANOVA), com posterior análise das médias pelo teste de Tukey a $5 \%$ de probabilidade com auxílio do programa Statistica 7.0, (STATSOFT, 2004).

\section{Resultados e discussão}

\subsection{Análise granulométrica}

Os resultados da análise granulométrica da matéria-prima utilizada na formulação dos snacks estão apresentados na Tabela 1.

As três matérias-primas estudadas diferiram significantemente quanto à granulometria, sendo que o grits de milho obteve uma classificação de tamanho de partículas entre 0,425 e 0,600 mm; o fubá mimoso, com partículas de 0,300 mm; e, por fim, o fubá mimoso fino, com partículas de granulometria inferiores a 250 $\mathrm{mm}$. Portanto, as amostras utilizadas encontram-se com parâmetros distintos de granulometria e com boa homogeneidade.

A granulometria das partículas do produto a ser extrusado pode afetar diretamente a textura e a uniformidade do extrusado. Matérias-primas com o tamanho das partículas variando de forma significativa resulta em um produto final com baixa qualidade (CARVALHO et al., 2012a), sendo de grande importância controlar a granulometria da matéria-prima a ser extrusada.

\subsection{Teor de amido e índice de expansão radial}

Os resultados da análise do teor de amido e índice de expansão radial estão apresentados na Tabela 2.

Os valores obtidos para amido não diferiram significantemente entre as amostras, uma vez que todas as matérias-primas eram provenientes da mesma safra

Tabela 1. Granulometria das matérias-primas para obtenção de snacks.

\begin{tabular}{ccccc} 
Mesh & $\begin{array}{c}\text { ABNT } \\
(\mathbf{m m})\end{array}$ & $\begin{array}{c}\text { Grits de milho } \\
\text { (\% retida) }\end{array}$ & $\begin{array}{c}\text { Fubá Mimoso } \\
\text { (\% retida) }\end{array}$ & $\begin{array}{c}\text { Fubá Mimoso Fino } \\
(\% \text { retida) }\end{array}$ \\
\hline 30 & 0,600 & $49,80 \pm 1,89^{\mathrm{A}}$ & $0,00 \pm 0,00^{\mathrm{B}}$ & $0,00 \pm 0,00^{\mathrm{B}}$ \\
40 & 0,425 & $42,04 \pm 2,05^{\mathrm{A}}$ & $7,60 \pm 1,15^{\mathrm{B}}$ & $0,32 \pm 0,21^{\mathrm{C}}$ \\
50 & 0,300 & $2,60 \pm 0,21^{\mathrm{C}}$ & $81,06 \pm 1,01^{\mathrm{A}}$ & $9,53 \pm 1,39^{\mathrm{B}}$ \\
60 & 0,250 & $0,40 \pm 0,52^{\mathrm{C}}$ & $5,16 \pm 1,38^{\mathrm{B}}$ & $17,10 \pm 0,55^{\mathrm{A}}$ \\
Fundo & ---- & $5,16 \pm 0,38^{\mathrm{B}}$ & $6,18 \pm 1,37^{\mathrm{B}}$ & $73,05 \pm 0,94^{\mathrm{A}}$ \\
\hline
\end{tabular}

Médias seguidas pela mesma letra na linha não diferem estatisticamente entre si pelo teste de Tukey a $5 \%$ de probabilidade. 
Influência da granulometria da matéria-prima na expansão de extrusados de milho

MIKALOUSKI, F. B. S. et al.

Tabela 2. Teor de amido nas matérias-primas dos extrusados e índice de expansão dos snacks.

\begin{tabular}{cccc} 
Variáveis & Grits & Fubá Mimoso & Fubá Mimoso Fino \\
Índice de Expansão & $4,73^{\mathrm{A}} \pm 0,31$ & $4,50^{\mathrm{A}} \pm 0,17$ & $3,99^{\mathrm{B}} \pm 0,27$ \\
Amido \% & $70,13^{\mathrm{A} \pm} 1,03$ & $71,62^{\mathrm{A}} \pm 1,11$ & $70,13^{\mathrm{A} \pm 1,08}$ \\
\hline
\end{tabular}

Médias seguidas pela mesma letra na linha não diferem estatisticamente entre si pelo teste de Tukey a 5\% de probabilidade.

Tabela 3. Resultado das médias para a dureza dos três diferentes snacks produzidos.

\begin{tabular}{cccc} 
Variáveis & Grits & Fubá Mimoso & Fubá Mimoso Fino \\
Dureza média (g.f.) & $950^{\mathrm{A}} \pm 230$ & $570^{\mathrm{B}} \pm 140$ & $370^{\mathrm{C}} \pm 90$ \\
\hline
\end{tabular}

Médias seguidas pela mesma letra na linha não diferem estatisticamente entre si pelo teste de Tukey a $5 \%$ de probabilidade.

de milho. Altos teores de amido estão relacionados a altos valores de índice de expansão utilizando matériaprima com baixo teor de lipídios e baixa umidade na hora da extrusão, pois lipídios e alta umidade reduzem a quantidade de amido gelificado na massa (CAPRILES e ARÊAS, 2012).

A grande expansão em snacks extrusados é desejada, pois estão relacionadas com a textura do produto. Extrusados com grande expansão são mais crocantes, segundo Capriles e Arêas (2011). Outro fator que contribui para a expansão dos extrusados a partir do grits do milho é o baixo teor de fibras, uma vez que, de acordo com Pai et al. (2009), a presença de fibras prejudica a expansão dos extrusados.

Os resultados obtidos para o índice de expansão radial foram de 3,99 a 4,73, inferiores aos encontrados por Silva et al. (2011), que observaram índices 11,49 para cereal matinal extrusado de mandioca enriquecido com concentrado proteico de soro de leite, e por Carvalho et al. (2012a), que encontraram valores variando de 5,35 a 14,83.

Segundo Carvalho et al. (2012b), a expansão na extrusão de produtos ricos em amido é inversamente proporcional à umidade do material a ser extrusado, ou seja, o aumento da umidade pode mudar a estrutura molecular e reduzir a viscosidade elástica, consequentemente reduzindo a expansão dos snacks.

Neste trabalho, as amostras foram condicionadas para umidade de $20 \%$ (por meio da adição de água, descontado o teor inicial de umidade de cada matériaprima), sendo esse um fator para os baixos valores obtidos no índice de expansão. Entretanto, como o objetivo era comparar as diferentes granulometrias, este fator manteve-se constante para todas as amostras, não interferindo no resultado.

\subsection{Análise da textura}

Segundo Capriles e Arêas (2012), a dureza em snacks é o atributo mais importante para sua aceitação; baixos valores de dureza resultam em alta crocância, atributo altamente importante para os consumidores.

Os valores obtidos para a dureza dos snacks estão apresentados na Tabela 3 e variaram de 270 g.f.
Tabela 4. Pontuação dos snacks de acordo com o teste de ordenação de preferência.

$\begin{array}{cccc}\text { Amostras } & \text { Grits } & \text { Fubá Mimoso } & \text { Fubá M. Fino } \\ \text { Preferência } & 99 & 90 & 111\end{array}$

a 1360 g.f. Pode-se observar que a dureza dos snacks está diretamente relacionada à granulometria da matériaprima, ou seja, quanto menor o tamanho das partículas menor a dureza do produto. Resultados obtidos por Mendonça et al. (1998) para snacks comerciais de milho extrusados a partir do grits variaram de 757,43 g.f. a 1210,17 g.f., valores no mesmo intervalo de confiança $(p<0,05)$ dos encontrados neste trabalho. No entanto, Oliveira et al. (2013) encontraram valores para extrusados com grits de milho, casca e semente de uva variando entre 2100 g.f. e 3480 g.f., que foram significativamente superiores aos encontrados neste trabalho, uma vez que a adição de fontes de fibras está forte e positivamente correlacionada ao aumento da dureza dos extrusados.

\subsection{Análise sensorial}

A Tabela 4 apresenta a pontuação obtida por cada amostra no teste de ordenação de preferência.

Utilizando a tabela de Newell e Mac Farlane $(\alpha=5 \%)$, obteve-se a mínima diferença significativa entre os totais de ordenação. A mínima diferença significativa para a análise com 50 provadores é de 24, logo, como a maior diferença entre as pontuações foi de 21 (fubá mimoso fino x fubá mimoso), é possível afirmar que não houve diferença significativa na aceitação dos snacks em função da variação da granulometria da matéria-prima.

De acordo com Annahas et al. (2013), em trabalho com snack adicionado de linhaça, os autores relatam que a variação de textura do extrusado não apresenta obrigatoriamente relação direta com a aceitação. Isto se deve ao fato de que alguns consumidores preferem a textura mais firme, enquanto outros preferem a mais macia.

De forma geral, com os resultados obtidos, foi possível observar que as matérias-primas utilizadas apresentaram-se diferentes na granulometria, porém o teor de amido presente em cada amostra se apresentou 
Influência da granulometria da matéria-prima na expansão de extrusados de milho

MIKALOUSKI, F. B. S. et al.

semelhante, pois todas as amostras derivam do milho limpo, degerminado e moído. A partir dos resultados obtidos, foi comprovada a influência da granulometria no índide de expansão pelas amostras que se apresentaram significativamente diferentes $(p<0,05)$, e também na textura dos snacks. Porém, a divergência nas características físicas dos snacks não ocasionou uma diferença signifcativa na preferência sensorial do produto. Isto se deve, principalmente, às diferenças de preferência entre os consumidores com relação à textura dos produtos.

\section{Conclusões}

A utilização do fubá em substituição ao grits de milho na produção de extrusados se mostrou eficaz, uma vez que, mesmo gerando alterações fisicas nos produtos finais, estas não interferiram na aceitação pelo consumidor, sendo uma alternativa para redução de custos para produtores de snacks extrusados de milho.

\section{Referências}

ASSOCIAÇÃO BRASILEIRA DAS INDÚSTRIAS MOAGEIRAS DE MILHO - ABIMILHO. Manual de Amostragem, Métodos Físico-Químicos e Microbiológicos para Análise de Produtos Derivados de Milho. Apucarana: ABIMILHO, 2003.

ANNAHAS, A. F. K.; MORAIS, D. R.; JOIA, B. M.; AGUIAR, A. C.; VISENTAINER, J. V.; MONTEIRO, A. R. G. Incorporation of omega-3 on an extruded snack with golden flaxseed. Journal of Food Science and Engineering, Essex, v. 3, n. 6, p. 317-322, 2013.

CAMARGO, R.; FONSECA, H.; PRADO FILHO, L. G.; ANDRADE, M. O.; CANTARELLI, P. R.; OLIVEIRA, A. J.; GRANER, M.; CARUSO, J. G. B.; NOGUEIRA, J. N.; LIMA, U. A.; MOREIRA, L. S. Tecnologia dos Produtos Agropecuários: Alimentos. Piracicaba: Nobel, 1984

CAPRILES, V. D.; ARÊAS, J. A. G. Avaliação da Qualidade Tecnológica de snacks obtidos por extrusão de grão integral de amaranto ou de farinha de amaranto desengordurada e suas misturas com fubá de milho. Brazilian Journal of Food Technology, Campinas, v. 15, n. 1, p. 21-29, 2012. http://dx. doi. org/10.1590/S1981-67232012000100003

CAPRILES, V. D.; ARÊAS, J. A. G. Redução da razão comprimento/diâmetro da extrusora e aumento da aceitabilidade de snacks à base de amaranto. Brazilian Journal of Food Technology, Campinas, v. 14, n. 1, p. 19-26, 2011. http://dx. doi. org/10.4260/BJFT2011140100003

CARVALHO, A. V.; BASSinelLO, P. Z.; MATTIETTO, R. A.; CARVALHO, R. N.; RIOS, A. O.; SECCADIO, L. L. Processamento e caracterização de snack extrudado a partir de farinhas de quirera de arroz e de bandinha de feijão. Brazilian Journal of Food Technology, Campinas, v. 15, n. 1, p. 72-83, 2012a. http:// dx.doi.org/10.1590/S1981-67232012000100008
CARVALHO, A. V.; RIOS, A. O.; BASSINELLO, P. Z.; SECCADIO, L. L. Otimização dos parâmetros tecnológicos para produção de snack extrudado a partir de arroz e feijão. Alimentação e Nutrição, Araraquara, v. 23, n. 3, p. 443-452, 2012b.

DISCHSEN, A. E.; MONTEIRO, A. R. G.; FUKUDA, G. T.; MARQUES, D. R. Development of a breakfast cereal using waste from cassava processing industry. Acta Scientiarum. Technology, Maringá, v. 35, n. 1, p. 157-161, 2013.

GONÇALVES, R. A.; SANTO, J. P. S.; TOME, P. H. F.; PEREIRA, R. G. F. A.; ASCHERIA, J. L. R.; ABREU, C. M. P. Rendimento e composição química de cultivares de milho em moagem a seco e produção de grifts. Ciência e Agrotecnologia, Lavras, v. 27, n. 3, p. 643-650, 2003.

MENDONÇA, S.; KARAM, L. B.; GROSSMANN, M. V. E. Análise instrumental de textura e sua correlação com a avaliação sensorial em "snacks" comerciais. In: CONGRESSO BRASILEIRO DE CIÊNCIA DE TECNOLOGIA DE ALIMENTOS, 16., 1998. Rio e Janeiro. Anais... Rio de Janeiro: SBCTA, 1998. p. 84-87. (v. 1).

MERCIER, C.; LINKO, P.; HARPER, J. M. Extrusion Cooking. 2. ed. St. Paul: American Association of Cereal Chemists, 1998. $199 \mathrm{p}$.

MONTEIRO, A. R. G.; CESTARI, L. A. Análise Sensorial de Alimentos: Testes Afetivos, Discriminativos e Descritivos. Maringá: EDUEM, 2013. v. 1, 53 p.

OLIVEIRA, D. M.; MARQUES, D. R.; KWIATKOWSKI, A.; MONTEIRO, A. R. G.; CLEMENTE, E. Sensory analysis and chemical characterization of cereal enriched with grape peel and seed flour. Acta Scientiarum. Technology, Maringá, v. 35, n. 3, p. 427-431, 2013.

PAI, D. A.; BLAKE, A. R.; HAMAKER, B. R.; CAMPANELLA, O. $\mathrm{H}$. Importance of extensional rheological properties on fiberenriched corn extrudates. Journal of Cereal Science, London, v. 50, n. 2, p. 227-234, 2009.

SCHMIELE, M. Caracterização das Frações com Diferentes Granulometrias de Milho Dentado e Duro e Avaliação na Qualidade de Extrudados Expandidos. 2009. 259 f. Dissertação (Mestrado em Tecnologia de Alimentos)-Faculdade de Engenharia de Alimentos, Universidade Estadual de Campinas, Campinas, 2009.

SILVA, P. A.; ASSIS, G. T.; CARVALHO, A. V.; SIMÕES, M. G. Desenvolvimento e caracterização de cereal matinal extrudado de mandioca enriquecido com concentrado proteico de soro de leite. Brazilian Journal of Food Technology, Campinas, v. 14, n. 4, p. 260-266, 2011. http://dx.doi.org/10.4260/ BJFT2011140400031

STATSOFT. Statística 7.0 software. Tucksa: StatSoft, Inc., 2004.

THAKUR, S.; SAXENA, D. C. Formulation of extruded snack food (gum based cerealpulse blend): optimization of ingredients levels using response surface methodology. Lebensmittel Wissenschaft und Technologie, London, v. 33, n. 5, p. 354-361, 2000. 